\title{
Clinical and Microbiological Outcomes of Er:YAG Laser Used as an Adjunct in Non-Surgical Periodontal Therapy: A Randomized Clinical Trial
}

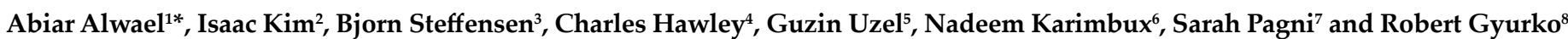

${ }^{1}$ DDS, MS, Periodontist and Implant Dentistry Fellowship, Tufts University School of Dental Medicine, Ministry of Health, Kuwait

${ }^{2} \mathrm{DMD}$, Periodontist, Private Practice in Massachusetts, Boston

${ }^{3}$ DDS, MS, PhD, Professor and Chair, Department of Periodontology, Tufts University School of Dental Medicine, Boston

${ }^{4} \mathrm{DDS}, \mathrm{MS}$, PhD, Adjunct Professor, Department of Periodontology, Tufts University School of Dental Medicine, Boston

${ }^{5}$ DMD, DMSc, Assistant Professor Department of Periodontology, Tufts University School of Dental Medicine, Boston

${ }^{6} \mathrm{DMD}, \mathrm{MMSc}$, Associate Professor, Department of Periodontology and Dean of Tufts University School of Dental Medicine, Boston

${ }^{7} \mathrm{MPH}, \mathrm{PhD}$, Assistant Professor, Department of Public Health and Community Services, Tufts University School of Dental Medicine, Boston

${ }^{8} \mathrm{DMD}$, PhD Associate Professor Department of Periodontology, Tufts University School of Dental Medicine, Boston

${ }^{\star}$ Corresponding author: Dr. Abiar Alwael, Ministry of Health, Kuwait; Tel: +965-66664147; Email: abiar_alwael@yahoo.com

Received: February 02, 2021; Accepted: February 18, 2021; Published: February 22, 2021

\begin{abstract}
Introduction: Er:YAG laser has the potential to reach areas of the periodontal pocket that are not accessible with traditional scaling and root planing (SRP). However the clinical and microbiological additional benefits of Er:YAG lasers as an adjunct to conventional SRP are not well documented.

Objective: Evaluate the efficacy of Er:YAG laser as an adjunct in non-surgical periodontal therapy (NSPT).

Materials and methods: A prospective randomized, controlled, split mouth single blinded clinical trial was performed on eleven participants diagnosed with moderate to severe periodontitis with probing depths (PD) $\geq 5 \mathrm{~mm}$. One quadrant received SRP with ultrasonic and hand instrumentation only, while in the other quadrant Er:YAG was used in addition to SRP. Clinical data were collected at baseline, 6 weeks and 3 months following SRP+/-Er:YAG. Microbial samples were collected, analyzed numerically, and cultured for a panel of periodontal pathogens at baseline and 3 months.

Results: In sites with $5 \mathrm{~mm}$ probing depths or more, the SRP group had an initial mean PD of $5.9 \pm 1.1 \mathrm{~mm}$ which decreased to $4.5 \pm 1.7 \mathrm{~mm}$ at 6 weeks and remained the same at 3 months. The SRP+Er:YAG group had an initial mean PD of $6.0 \pm 1.4 \mathrm{~mm}$ that decreased to $4.6 \pm 2.0 \mathrm{~mm}$ and to $4.6 \pm 1.8 \mathrm{~mm}$ at 6 weeks and 3 months respectively. The SRP group had an initial mean BOP of $92.7 \%$ which decreased to mean of $64.6 \%$ and $61.5 \%$ at the 6 weeks and 3 months respectively. The SRP+Er:YAG group had an initial mean BOP of $80.9 \%$. BOP decreased to $58.4 \%$ and $43.8 \%$ at 6 weeks and 3 months respectively. There were no statistically significant differences between the SRP and SRP+Er:YAG group in clinical parameters and bacterial counts. There was an overall decrease in the percentage of the subgingival cultivable microflora at 3 months in both groups. There were no statistically significant differences between the SRP and SRP+Er:YAG group with respect to clinical changes, bacterial counts, and cultivability profiles.
\end{abstract}

Conclusion: Within the study limitations, the use of Er:YAG laser when used as an adjunct to SRP conferred no additional clinical and microbial benefits.

\section{Introduction}

Er:YAG (erbium-doped yttrium aluminum garnet) laser is one of the most frequently used lasers in non-surgical and surgical periodontal therapy. Er:YAG lasers emit infrared light at $2940 \mathrm{~nm}$ which is highly absorbed by water molecules, resulting in instant vaporization. Er:YAG laser light is well absorbed by all biological tissues with high water content and it can be used for soft tissue ablation and bacterial killing [1]. Er:YAG laser light is also absorbed by hydroxyapatite, thus making it suitable for ablation and calculus removal [2]. These properties are advantageous in periodontal therapy where removal of plaque and calculus is traditionally done by scaling and root planing (SRP).
Er:YAG is a suitable choice for effective calculus removal. Its wavelength and the use of water spray surface coolant effectively remove smear layers, calculus, and necrotic cementum from root surfaces without inducing tissue damage. The use of Er:YAG laser has demonstrated better wound healing, decreased swelling and scarring, less pain and better patient tolerance compared to conventional nonsurgical and surgical methods [3].

Several other studies have shown improved periodontal clinical parameters after laser therapy. Schwarz et al. showed that laser therapy resulted in significant reduction in bleeding on probing and gains in clinical attachment levels compared to scaling and root planning alone. The clinical attachment gain in the laser group was maintained for more than 2 years [4]. Ando et al. concluded from an in vitro 
study that the Er:YAG laser has bactericidal effect at low energy levels [5] and Ishikawa et al. reported that Er:YAG laser may provide an antimicrobial advantage compared to conventional mechanical periodontal therapy [1]. The laser degrades and removes bacterial endotoxins without producing a smear layer [1]. However, a recent review of the literature was inconclusive regarding the clinical effects and microbiological outcomes of Er:YAG lasers when used as an adjunct to conventional scaling and root planing [3].

While scaling and root planing results in significant reduction of tissue inflammation [6], several studies have demonstrated that there is a limitation in the efficacy of curettes during scaling and root planing in periodontal pockets that are $5 \mathrm{~mm}$ or more $[7,8]$. Thus, there is clearly a need for developing effective methods of plaque and calculus removal in deep pockets and hard-to access locations. In addition, the evaluation of Er-YAG laser as an adjunct to SRP is needed, as the two treatments are not mutually exclusive.

The purpose of this study was to evaluate the efficacy of Er:YAG laser as an adjunct to scaling and root planing in non-surgical periodontal therapy on probing depth, clinical attachment levels, gingival bleeding and microbial colonization patterns.

\section{Materials and Methods}

\section{Enrollment of Participants}

This prospective randomized, controlled, split mouth single blinded clinical trial was conducted at Tufts University School of Dental Medicine (TUSDM). The protocol was approved by the Tufts University Medical Center Institutional Review Board (IRB \#12321). Participants were patients enrolled for treatment in the Post-graduate Periodontology Clinic at TUSDM.

Potential participants were informed about the study and provided with ample time to ask any questions on study participation. Patients who decided to participate then provided informed. A copy of the informed consent form (ICF) was given to the participant. Demographic information was collected and a medical history was obtained.

\section{Inclusion Criteria}

To qualify for the study, each subject had to have all single rooted permanent maxillary and mandibular teeth from the central incisors to the second premolars, a recent diagnosis of moderate to severe chronic periodontitis [8] and a full mouth and vertical bite-wing series of diagnostic radiographs exposed at TUSDM within 6 months preceding entry of the study.

\section{Exclusion Criteria}

Participants who did not speak or write English were excluded from the study. Participants who had received mechanical debridement or any other professional periodontal therapy within 6 months prior to entering the study, who presented with significant chronic oral soft tissue pathologies, who presented with fixed or removable appliances partial dentures, who were current smokers, who ordinarily required prophylactic antibiotics prior to dental procedures, or who had taken systemic antibiotic medications within the previous 6 were excluded from the study.

Participants with uncontrolled chronic systemic conditions or diseases such as diabetes mellitus ( $\mathrm{HbA} 1 \mathrm{C}>7 \%$ ), with immunological disorders, with known drug allergies or known adverse effects following the use of oral hygiene products, and who might be pregnant or lactating were excluded from the study.

Also, teeth with Miller grade III mobility or teeth with hopeless prognosis [9] indicated for extraction were excluded from the study.

\section{Clinical Measurements}

Using radiographs and the results of a complete periodontal examination, the diagnosis of generalized moderate to severe chronic periodontitis was confirmed, The examiner (I.K.) was previously calibrated to $>90 \%$ accuracy in repeat probing depth measurements using the UNC probe [10]. Clinical measurements were taken at baseline and at 6 weeks and 3 months post treatment. The examiner was blind to all treatment assignments.

Two quadrants in each subject with single rooted teeth that demonstrated periodontal probings $\geq 5 \mathrm{~mm}$ were selected for the trial. A computer-generated randomization list was used to determine which quadrant would receive scaling and root planing only and which quadrant would receive SRP supplemented with the Er:YAG laser therapy.

\section{Microbial Sampling and Transport}

Before treatment, baseline microbial samples were obtained on single rooted, non-furcated teeth with $5-9 \mathrm{~mm}$ probing depths and bleeding on probing. The deepest two sites in each quadrant were chosen for microbial sampling giving a total of 4 microbial samples per participant. These same sites would be sampled again for microbial analysis post- treatment at the scheduled 3 month follow-up visit.

After air drying and isolation with cotton rolls and careful removal of supra-gingival plaque, one sterile, absorbent paper point, (Johnson \& Johnson, East Windsor, NJ), was advanced into each of the selected periodontal sites for 10 seconds [11]. After removal, the two paper points per quadrant were pooled together in a glass vial of $2.0 \mathrm{~mL}$ anaerobically prepared viability medium Gothenburg anaerobic (VMGA) III transport medium [10]. Subgingival samples were then transported within 24 hours for microbial analysis to the Oral Microbiology Testing Service (OMTS) Laboratory located at the Temple University Kornberg School of dentistry. All laboratory microbiologic procedures were performed by OMTS personnel who were unaware of the participants' overall health status, clinical diagnosis, and site specific conditions or that participants were part of a clinical trial [10].

Putative periodontal pathogens examined for in this study include Aggregatibacter actinomycetemcomitans, Porphyromonas gingivalis, Tannerella forsythia, Prevotella intermedia/nigrescens, Parvimonas micra, Fusobacterium nucleatum, Campylobacter rectus, Streptococcus constellatus, Streptococcus intermedius, Staphylococcus aureus, Enterococcus faecalis, Gram-negative enteric rods/pseudomonads, 
and Candida species [10]. The specimen vials were heated to $35^{\circ} \mathrm{C}$ $[10,12]$. The plaque samples were dispersed from the paper points using a vortex mixer for 45 seconds in Möller's viability medium Gothenburg I anaerobic dispersion solution comprised of pre-reduced, anaerobically sterilized $0.25 \%$ tryptose, $0.25 \%$ thiotone E peptone, and $0.5 \%$ sodium chloride. The same medium was used to create a 10 -fold dilutions of the bacterial suspensions [10]. Then, $0.1 \mathrm{~mL}$ dilution aliquots were spread with a sterile bent-glass rod onto non- selective enriched Brucella blood agar (EBBA) primary isolation plates [10]. EBBA was comprised of $4.3 \%$ Brucella agar supplemented with $0.3 \%$ bacto-agar, $5 \%$ defibrinated sheep blood, $0.2 \%$ hemolyzed sheep red blood cells, $0.0005 \%$ hemin, and $0.00005 \%$ menadione. EBBA plates were incubated at $35^{\circ} \mathrm{C}$ for 7 days in an anaerobic chamber containing $85 \% \mathrm{~N} 2,10 \% \mathrm{H} 2$, and $5 \% \mathrm{CO}_{2}$, EBBA plates were used to determine the composition of predominant cultivable microbial species using established OMTS methods [10,11].

Total anaerobic viable counts were made on non-selective EBBA primary isolation plates. Porphyromonas gingivalis, Tannerella forsythia, Prevotella intermedia/nigrescens, Parvimonas micra, Fusobacterium nucleatum, Campylobacter rectus, Streptococcus constellatus, Streptococcus intermedius, Staphylococcus aureus, Enterococcus faecalis were distinguished on EBBA using established microbial identification OMTS protocols [10]. Aggregatibacter actinomycetemcomitans, Gram-negative enteric rods, pseudomonads, and Candida species were quantitated on selective trypticase soybacitracin-vancomycin (TSBV) agar plates that were incubated at $35^{\circ} \mathrm{C}$ for 3 days in air plus $5 \%$ CO2 [10]. The proportional recovery of each test species was ascertained for each individual by calculating the percentage of positive test species colony forming units relative

Table 1: Demographics of the study population.

\begin{tabular}{|c|c|c|}
\hline \multicolumn{3}{|c|}{ Parameters } \\
\hline Age (ye & mean $\pm S D$ ) & $48 \pm 16$ \\
\hline \multicolumn{3}{|c|}{ Gender n (\%) } \\
\hline • & Male & $6(54.6 \%)$ \\
\hline • & Female & $5(45.5 \%)$ \\
\hline \multicolumn{3}{|c|}{ Race n (\%) } \\
\hline • & Native Americans & $3(27.2 \%)$ \\
\hline - & Asian & $3(27.2 \%)$ \\
\hline • & Black/African American & $2(18.18 \%)$ \\
\hline • & White/Non- Hispanic & $3(27.2 \%)$ \\
\hline Total & & 11 \\
\hline
\end{tabular}

to total subgingival anaerobic viable counts as determined on nonselective EBBA primary isolation plates [10].

\section{Treatment}

Scaling and root planing was performed with an ultrasonic scaler (Varios 360 LUX Model: NE149) and hand scalers and Gracey curettes under local anesthesia using $2 \%$ lidocaine $+1: 100000$ epinephrine.

Er:YAG laser treatment was delivered with AdvErL Evo Er:YAG Laser for Dentistry (Morita Model: MEY-1-A), with PS600TS tip using the manufacturer's recommended settings $(70 \mathrm{~mJ}, 25$ pulses per second, PPS). The laser tip was inserted into the periodontal pockets measured prior treatment with $\mathrm{PD} \geq 5 \mathrm{~mm}$ and was moved repeatedly in an apico-coronal direction without touching the root for 10 seconds.

Participants were given a prescription of $0.12 \%$ chlorhexidine (Peridex) mouthwash to use for 6 weeks after treatment. One operator (A.A) performed all scaling and root planing and Er:YAG laser treatments.

\section{Statistical Analysis}

Descriptive statistics (means and standard deviations for continuous items, counts and percentages of categorical items) were calculated.

Differences in probing depth reduction or attachment gain between laser treatment and control groups were analyzed with a nested mixed effects linear regression model. Normality of the data was assessed graphically. Statistical significance between the two groups' gingival bleeding index was determined with a generalized estimating equation model. Differences in microbial load was investigated with the Wilcoxon signed-rank test. P-values less than 0.05 were considered statistically significant. The software Stata 13.1 (StataCorp LLC, College Station, TX) was used for the analysis.

\section{Results}

Seventeen study volunteers were screened and 11 participants (6 males and 5 females) were enrolled in this prospective, randomized single-blinded clinical trial. All 11 study participants complied fully with all study procedures and follow-up visits. The mean age of the study population was $48 \pm 16$ years. Table 1 shows the demographic background of the study population.

Clinical parameters (PD, CAL and BOP) were improved after treatment in both the SRP and SRP+Er:YAG laser group (Table 2). However, there were no significant differences between the SRP and the SRP + Er:YAG groups at 3 months $(\mathrm{P}=0.08)$.

Table 2: Mean probing depths (PD, $\mathrm{mm}$ ), clinical attachment levels (CAL, $\mathrm{mm}$ ), and bleeding on probing (BOP, \%) of sites with baseline probing depth of $\geq 5 \mathrm{~mm}$ in SRP only (Control) and SRP + Er:YAG (Test) groups at baseline, 6 weeks and 3 months for all study participants.

\begin{tabular}{|c|c|c|c|c|c|c|}
\hline \multirow[t]{2}{*}{ Time } & \multicolumn{2}{|c|}{ Baseline } & \multicolumn{2}{|c|}{6 Weeks } & \multicolumn{2}{|c|}{3 Months } \\
\hline & $\begin{array}{c}\text { SRP Only } \\
(\text { Mean } \pm \text { SD })\end{array}$ & $\begin{array}{l}\text { SRP + Er:YAG laser } \\
(\text { Mean } \pm \text { SD })\end{array}$ & $\begin{array}{c}\text { SRP Only } \\
(\text { Mean } \pm \text { SD })\end{array}$ & $\begin{array}{l}\text { SRP + Er:YAG laser } \\
(\text { Mean } \pm \text { SD })\end{array}$ & $\begin{array}{c}\text { SRP Only } \\
(\text { Mean } \pm \text { SD })\end{array}$ & $\begin{array}{c}\text { SRP + Er:YAG laser } \\
(\text { Mean } \pm \text { SD })\end{array}$ \\
\hline Mean PD & $5.9 \pm 1.1$ & $6.0 \pm 1.4$ & $4.5 \pm 1.7$ & $4.6 \pm 2.0$ & $4.7 \pm 1.6$ & $4.6 \pm 1.8$ \\
\hline Mean CAL & $6.2 \pm 1.3$ & $6.3 \pm 1.5$ & $4.9 \pm 2.5$ & $5.0 \pm 2.4$ & $5.3 \pm 2.3$ & $5.0 \pm 2.2$ \\
\hline Mean BOP & $92.7 \%$ & $80.9 \%$ & $64.6 \%$ & $58.4 \%$ & $61.5 \%$ & $43.8 \%$ \\
\hline
\end{tabular}

$\mathrm{SD}=$ Standard deviation. 
Table 3: Comparison between the mean percentage in the cultivable microflora between baseline and 3 months for the SRP and SRP+Er:YAG groups.

\begin{tabular}{|c|c|c|c|c|c|c|c|c|}
\hline \multirow[t]{3}{*}{ Bacteria (\%) } & \multicolumn{4}{|c|}{ SRP } & \multicolumn{4}{|c|}{ SRP+Er:YAG } \\
\hline & \multicolumn{2}{|c|}{ Baseline } & \multicolumn{2}{|c|}{3 Months } & \multicolumn{2}{|c|}{ Baseline } & \multicolumn{2}{|c|}{3 Months } \\
\hline & Mean $\pm \mathrm{SD}^{*}$ & Frequency** & \begin{tabular}{|l|} 
Mean \pm SD \\
\end{tabular} & Frequency & \begin{tabular}{|l|} 
Mean \pm SD \\
\end{tabular} & Frequency & Mean \pm SD & Frequency \\
\hline $\begin{array}{c}\text { A. actinomycetemcomitans } \\
\text { (Aa) }\end{array}$ & $0 \pm 0$ & $0 / 11$ & $0 \pm 0$ & $0 / 11$ & $0 \pm 0$ & $0 / 11$ & $0 \pm 0$ & $0 / 11$ \\
\hline $\begin{array}{c}\text { Porphyromonas gingivalis } \\
(\mathrm{Pg})\end{array}$ & $0.3 \pm 0.8$ & $1 / 11$ & $0 \pm 0$ & $0 / 11$ & $0.3 \pm 0.9$ & $1 / 11$ & $0 \pm 0$ & $0 / 11$ \\
\hline Tannerella forsythia (Tf) & $3.4 \pm 8.9$ & $3 / 11$ & $0.9 \pm 2.2$ & $2 / 11$ & $1.1 \pm 2.4$ & $3 / 11$ & $0.5 \pm 1.2$ & $2 / 11$ \\
\hline Prevotella intermedia $(P i)$ & $3.5 \pm 3.2$ & $10 / 11$ & $2.7 \pm 6.1$ & $5 / 11$ & $6.0 \pm 8.6$ & 9/11 & $1.2 \pm 2.5$ & $3 / 11$ \\
\hline $\begin{array}{l}\text { Fusobacterium nucleatum } \\
\text { (Fn) }\end{array}$ & $8.3 \pm 6.9$ & $10 / 11$ & $4.5 \pm 4.3$ & $8 / 11$ & $7.9 \pm 6.9$ & $11 / 11$ & $4.0 \pm 7.0$ & $7 / 11$ \\
\hline Parvimonas micra $(\mathrm{Pm})$ & $6.5 \pm 5.4$ & $10 / 11$ & $6.2 \pm 4.9$ & $11 / 11$ & $7.3 \pm 6.7$ & $10 / 11$ & $4.9 \pm 3.5$ & $10 / 11$ \\
\hline Campylobacter rectus $(\mathrm{Cr})$ & $0 \pm 0$ & $0 / 11$ & $0.01 \pm 0$ & $1 / 11$ & $0 \pm 0$ & $0 / 11$ & $0 \pm 0$ & $0 / 11$ \\
\hline $\begin{array}{c}\text { Streptococcus constellatus } \\
(\mathrm{Sc})\end{array}$ & $0 \pm 0$ & $0 / 11$ & $0.7 \pm 2.2$ & $1 / 11$ & $0 \pm 0$ & $0 / 11$ & $0 \pm 0$ & $0 / 11$ \\
\hline $\begin{array}{l}\text { Streptococcus intermedius } \\
\text { (Si) }\end{array}$ & $0.3 \pm 0.9$ & $1 / 11$ & $0 \pm 0$ & $0 / 11$ & $0 \pm 0$ & $0 / 11$ & $1.8 \pm 6.0$ & $1 / 11$ \\
\hline Enteric gram-negative rods & $0 \pm 0$ & $0 / 11$ & $0 \pm 0$ & $0 / 11$ & $0 \pm 0$ & $0 / 11$ & $0 \pm 0$ & $0 / 11$ \\
\hline Enterococcus faecalis (Ef) & $0 \pm 0$ & $0 / 11$ & $0 \pm 0$ & $0 / 11$ & $0 \pm 0$ & $0 / 11$ & $0 \pm 0$ & $0 / 11$ \\
\hline Staphylococcus aureus & $0 \pm 0$ & $0 / 11$ & $0 \pm 0$ & $0 / 11$ & $0 \pm 0$ & $0 / 11$ & $0 \pm 0$ & $0 / 11$ \\
\hline Candida species & $0 \pm 0$ & $0 / 11$ & $0 \pm 0$ & $0 / 11$ & $0 \pm 0$ & $0 / 11$ & $0 \pm 0$ & $0 / 11$ \\
\hline
\end{tabular}

* Mean of 11 participants; 2 sites analyzed and pooled in the same vial in each participant

** Frequency of detection in 11 participants

Microbiological data was expressed as percentage of the cultivable microflora before and 3 months after SRP or SRP+Er:YAG treatment. A. actinomycetemcomitans, Enteric gram negative rods, E. faecalis, $S$. aureus and Candida species were not detected in any participant at baseline or at the 3 months follow up visit in either the test and control groups in all participants. Porphyromonas gingivalis was detected in one participant only in both treatment groups (Table 3 ). When comparing baseline and 3 months data, there was an overall reduction in the percentage of cultivable microflora for Porphyromonas gingivalis, Tannerella forsythia, Prevotella intermedia, Fusobacterium nucleatum and Parvimonas micra in both SRP and SRP+Er:YAG laser groups. There was a slight increase in Campylobacter rectus and Streptococcus constellatus in the SRP group and in Streptococcus intermedius in the SRP+Er:YAG laser group from baseline to 3 months follow up in one participant only. However no statistically significant differences in percentage of the cultivable microflora were found when comparing SRP and SRP-Er:YAG lase treated groups. Since the data is not normally distributed, the Wilcoxon signed-rank test was used. There was no statistically significant difference in the change in any bacteria between baseline and 3 months follow up $(\mathrm{P}>0.05)$ (Table 4).

\section{Discussion}

The current literature is equivocal regarding the efficacy of Er:YAG laser as part of non-surgical periodontal therapy. The present prospective randomized single-blinded clinical trial was designed to better understand the efficacy of Er:YAG laser therapy on clinical periodontal parameters as well as microbial recolonization patterns in patients diagnosed with chronic moderate to severe chronic periodontitis.

Er:YAG laser in combination with SRP and ultrasonic instrumentation effectively reduced PD by $1.4 \mathrm{~mm}$ from baseline to 3 months in sites with $\mathrm{PD} \geq 5 \mathrm{~mm}$. This is comparable to results in a literature review by Cobb, who reported an average PD reduction of $1.29 \mathrm{~mm}$ in 4-6 $\mathrm{mm}$ sites [13]. However, we did not find statistically significant differences between SRP and SRP+Er:YAG treated pockets
Table 4: Comparison between the difference in the mean of the cultivable microflora between baseline and 3 months for the SRP and SRP+Er:YAG groups.

\begin{tabular}{|c|c|c|c|}
\hline Bacteria/Time & $\begin{array}{c}\text { SRP }^{\star} \\
(\text { mean } \pm \text { SD }) \\
(\text { Median/IQR })\end{array}$ & $\begin{array}{c}\text { SRP + Er:YAG }{ }^{*} \\
(\text { mean } \pm \text { SD }) \\
(\text { Median/IQR })\end{array}$ & $P$ value \\
\hline A. actinomycetemcomitans (Aa) & Not detected & Not detected & - \\
\hline Porphyromonas gingivalis $(\mathrm{Pg})$ & $\begin{array}{c}0.2 \pm 0.8 \\
0 / 0\end{array}$ & $\begin{array}{c}0.2 \pm 0.9 \\
0 / 0\end{array}$ & 0.32 \\
\hline Tannerella forsythia (Tf) & $\begin{array}{c}2.4 \pm 9.6 \\
0 / 2.1\end{array}$ & $\begin{array}{c}0.6 \pm 2.6 \\
0 / 0.7\end{array}$ & 0.42 \\
\hline Prevotella intermedia $(\mathrm{Pi})$ & $\begin{array}{c}0.7 \pm 6.4 \\
2 / 5.4\end{array}$ & $\begin{array}{c}4.7 \pm 7.9 \\
2 / 10\end{array}$ & 0.33 \\
\hline Fusobacterium nucleatum (Fn) & $\begin{array}{c}3.8 \pm 8.2 \\
4.2 / 6.5\end{array}$ & $\begin{array}{c}3.9 \pm 9.8 \\
3 / 9\end{array}$ & 0.32 \\
\hline Parvimonas micra (Pm) & $\begin{array}{l}0.3 \pm 8.4 \\
-0.9 / 11.7\end{array}$ & $\begin{array}{c}2.4 \pm 6.3 \\
0.1 / 8.3\end{array}$ & 0.53 \\
\hline Campylobacter rectus (Cr) & $\begin{array}{c}-0.009 \pm 0.03^{* *} \\
0 / 0\end{array}$ & $\begin{array}{c}0.00 \pm 0.00 \\
0 / 0\end{array}$ & 0.32 \\
\hline Streptococcus constellatus (Sc) & $\begin{array}{c}-0.66 \pm 2.2^{* *} \\
0 / 0 \\
\end{array}$ & $\begin{array}{c}0.00 \pm 0.00 \\
0 / 0 \\
\end{array}$ & 0.32 \\
\hline Streptococcus intermedius $(\mathrm{Si})$ & $\begin{array}{c}0.3 \pm 0.9 \\
0 / 0\end{array}$ & $\begin{array}{c}-1.8 \pm 6.03^{* *} \\
0 / 0\end{array}$ & 0.16 \\
\hline Enteric gram negative rods & Not detected & Not detected & - \\
\hline Enterococcus faecalis (Ef) & Not detected & Not detected & - \\
\hline Staphylococcus aureus & Not detected & Not detected & - \\
\hline Candida species & Not detected & Not detected & - \\
\hline
\end{tabular}

$\mathrm{SD}=$ Standard deviation IQR: Inter-quartile range.

${ }^{\star}$ Difference between baseline and 3 months data from 11 participants.

${ }^{*}$ Negative value indicates an increase from baseline to 3 months.

after 6 weeks and 3 months. Periodontal inflammation as measured by BOP also decreased in both of our treatment groups at 6 weeks and 3 months; however there was no significant difference between the SRP and SRP+Er:YAG groups. These observations are in line with previous studies [14-19].

Differences in the outcomes of published clinical trials on the effect of Er:YAG laser may be attributable to the various treatments 
the control groups have received: no treatment, SRP alone, ultrasonic scaling alone or a combination of SRP and ultrasonic; and whether the laser was used alone or in combination with other treatment modalities. A number of studies tested Er:YAG laser against SRP alone, and found no significant Er:YAG laser effect in terms of PD reduction at 3, 6 or 12 months [14-18]. On the other hand, Schwarz et al. [4] reported superior outcomes with Er:YAG laser alone compared to SRP in a randomized controlled split mouth clinical trial. Recently, Zhou et al. found statistically significant effect of Er:YAG laser when compared to SRP alone, albeit the effect was judged clinically minimally important by the authors [20]. Compared to ultrasonic debridement alone, adjunct application of Er:YAG laser induced significant PD reduction 12 months after treatment [19].

In comparison, our approach was to use the common standard of care (SRP combined with ultrasonic scaling) as control, and add Er:YAG laser as an adjunct. Findings presented here indicate that Er:YAG laser does not confer additional benefits in terms of pocket reduction or elimination of inflammation when used in combination with SRP and ultrasonic scaling. An important factor to be considered when comparing studies is various laser energy and pulse settings. In our study we applied $70 \mathrm{~mJ}$ at $25 \mathrm{~Hz}$, following manufacturer's recommendations. Other investigations employed different laser sources and different pulse settings: Schwarz et al. used $160 \mathrm{~mJ}$ at 10 $\mathrm{Hz}$ [4] and Zhou et al. used 50-100 $\mathrm{mJ}$ at $15-30 \mathrm{~Hz}$ [20].

We found an overall decrease of the subgingival microbial load at 3 months compared to baseline but that decrease was not significant. This is consistent with other in literature where the total number of the subgingival microbiota was reduced after therapy but the gramnegative species returned to baseline 3 - 6 months post therapy [21,22]. The literature is inconsistent when microbial outcomes were measured. Some studies have shown that there was no significant difference in terms of microbial outcomes between the SRP only group and SRP+Er:YAG laser groups $[14,18,19]$. One study found significantly greater reduction in A. actinomycetemcomitans, Porphyromonas gingivalis, Prevotella nigrescens and Tannerella forsythia at 6 and 12 months in the SRP + Er:YAG laser group [16]. In that study, patients were enrolled in a rigorous plaque-control program prior to laser treatment.

One of the limitations of our present study is that bacterial samples were collected only after 3 months. By that time the microbial load may have returned to its pre-treatment baseline [21]. Also, using bacterial cultures only enables us to detect live bacteria. Utilizing more sensitive approaches in the microbial analysis might have enabled us to detect more cultivable microflora. It cannot be excluded that the split mouth design might have resulted in translocation of bacteria between the test and control quadrants that influenced the clinical and microbial results. Increasing the sample size might have permitted us to detect treatment effects that did not reach statistical significance. Also, longer follow up periods could have added to our knowledge on the long-term efficacy of laser therapy.

Contradictory data in the literature may be due to heterogeneity in study design such as the different methods used for bacterial sampling and detection, the time it took for samples to be analyzed after they were obtained, different equipment and settings, the method and efficacy of SRP, the uneven root surface topography, the thickness of the subgingival biofilm and the timing and duration of laser therapy. It appears that both the laser type and settings impact the outcomes achieved in different studies. Further studies are needed to establish appropriate settings and the use of water coolant for periodontal nonsurgical therapy with the laser as monotherapy or as an adjunct to SRP [24-26].

Although patient-centered outcomes were not measured in this study, several participants reported less post-treatment sensitivity on the Er:YAG laser treated side. This coincides with studies that have shown significant reduction in dentin hypersensitivity following Er:YAG application [27,28]. Likewise, bleeding during instrumentation appeared to be less on the Er:YAG laser treated sites, suggestive of Er:YAG laser's hemostatic properties, which may lead to improved postoperative patient experience, particularly in those taking anticoagulants [2]. It is worth noting that none of the participants reported adverse outcomes. Thus, for future considerations, an evaluation of the posttreatment experience by the participants may add value to the results.

\section{Conclusion}

Scaling and root planing with or without Er:YAG laser treatment reduced periodontal inflammation as measured by probing depth reduction, gain in clinical attachment and decreased bleeding on probing. Within the limitations of this study, the use of Er:YAG laser as an adjunctive therapy to scaling and root planing in patients diagnosed with moderate to severe chronic periodontitis did not provide clinical and microbial benefit over a combination of ultrasonic and hand instrumentation.

\section{Acknowledgements}

Thomas Rams, DDS, MHS, PhD, Professor - Director of Oral Microbiology Testing Service Laboratory, Department of Periodontology and Oral Implantology, Kornberg School of Dentistry.

\section{Conflict of Interest}

The authors declare that they have no conflict of interests in this study.

\section{References}

1. Ishikawa I, A. Aoki, Takasaki AA (2004) Potential applications of Erbium:YAG laser in periodontics. J Periodontal Res 39(4): 275-85. [crossref]

2. Aoki A, et al. (2004) Lasers in nonsurgical periodontal therapy. Periodontol 2000, 36: 59-97. [crossref]

3. Cobb CM (2006) Lasers in periodontics: a review of the literature. J Periodontol 77(4): 545-64. [crossref]

4. Schwarz F, et al. (2001) Periodontal treatment with an Er: YAG laser compared to scaling and root planing. A controlled clinical study. J Periodontol 72(3): 361-7. [crossref]

5. Ando Y, et al. (1996) Bactericidal effect of erbium YAG laser on periodontopathic bacteria. Lasers Surg Med 19(2): 190-200. [crossref]

6. Caton J, et al. (1989) Effects of personal oral hygiene and subgingival scaling on bleeding interdental gingiva. J Periodontol 60(2): 84-90. [crossref]

7. Waerhaug J (1978) Healing of the dento-epithelial junction following subgingival plaque control. I. As observed in human biopsy material. J Periodontol 49(1): 1-8. [crossref] 
8. American Academy of Periodontology Task Force Report on the Update to the 1999 Classification of Periodontal Diseases and Conditions (2015) J Periodontol 86(7): 835-8. [crossref]

9. McGuire MK (1991) Prognosis versus actual outcome: a long-term survey of 100 treated periodontal patients under maintenance care. J Periodontol 62(1): 51-8. [crossref]

10. Polson AM, et al. (1997) Multi-center comparative evaluation of subgingivally delivered sanguinarine and doxycycline in the treatment of periodontitis. I. Study design, procedures, and management. J Periodontol 68(2): 110-8. [crossref]

11. Rams TE, Degener JE, van Winkelhoff AJ (2014) Antibiotic resistance in human chronic periodontitis microbiota. J Periodontol 85(1): 160-9. [crossref]

12. Rams TE, Listgarten MA, Slots J (1996) Utility of 5 major putative periodontal pathogens and selected clinical parameters to predict periodontal breakdown in patients on maintenance care. J Clin Periodontol 23(4): 346-54. [crossref]

13. Cobb CM, McCawley TK, Killoy WJ (1992) A preliminary study on the effects of the Nd:YAG laser on root surfaces and subgingival microflora in vivo. J Periodontol 63(8): 701-7. [crossref]

14. Yilmaz S, et al. (2013) Evaluation of the clinical and antimicrobial effects of the Er:YAG laser or topical gaseous ozone as adjuncts to initial periodontal therapy. Photomed Laser Surg 31(6): 293-8. [crossref]

15. Lopes BM, et al. (2010) Clinical and microbiologic follow-up evaluations after nonsurgical periodontal treatment with erbium:YAG laser and scaling and root planing. J Periodontol 81(5): 682-91. [crossref]

16. Rotundo R, et al. (2010) Lack of adjunctive benefit of Er:YAG laser in non-surgica periodontal treatment: a randomized split-mouth clinical trial. J Clin Periodontol 37(6): 526-33. [crossref]

17. Badran Z, et al. (2012) Clinical outcomes after nonsurgical periodontal therapy with an Er:YAG laser device: a randomized controlled pilot study. Photomed Laser Surg 30(7): 347-53. [crossref]
18. Yilmaz S, et al. (2012) Er:YAG laser versus systemic metronidazole as an adjunct to nonsurgical periodontal therapy: a clinical and microbiological study. Photomed Laser Surg 30(6): 325-30. [crossref]

19. Sanz-Sanchez I, et al. (2015) Microbiological effects and recolonization patterns after adjunctive subgingival debridement with Er:YAG laser. Clin Oral Investig

20. Zhou X, et al. (2019) Efficacy of Er:YAG laser on periodontitis as an adjunctive nonsurgical treatment: A split-mouth randomized controlled study. J Clin Periodontol 46(5): 539-547. [crossref]

21. Haffajee AD, et al. (1997) Clinical and microbiological features of subjects with adult periodontitis who responded poorly to scaling and root planing. J Clin Periodontol 24(10): 767-76. [crossref]

22. Slots J, et al. (1979) Periodontal therapy in humans. I. Microbiological and clinical effects of a single course of periodontal scaling and root planing, and of adjunctive tetracycline therapy. J Periodontol 50(10): 495-509. [crossref]

23. Schwarz F, et al. (2003) Clinical evaluation of an Er:YAG laser combined with scaling and root planing for non-surgical periodontal treatment. A controlled, prospective clinical study. J Clin Periodontol 30(1): 26-34. [crossref]

24. Mousques T, Listgarten MA, Phillips RW (1980) Effect of scaling and root planing on the composition of the human subgingival microbial flora. J Periodontal Res 15(2): 144-51. [crossref]

25. Magnusson I, et al. (1984) Recolonization of a subgingival microbiota following scaling in deep pockets. J Clin Periodontol 11(3): 193-207. [crossref]

26. Schwarz F, et al. (2003) In vivo and in vitro effects of an Er:YAG laser, a GaAlAs diode laser, and scaling and root planing on periodontally diseased root surfaces: a comparative histologic study. Lasers Surg Med 32(5): 359-66. [crossref]

27. Schwarz F, et al. (2002) Desensitizing effects of an Er:YAG laser on hypersensitive dentine. J Clin Periodontol 29(3): 211-5. [crossref]

28. Ehlers V (2012) Clinical comparison of gluma and Er:YAG laser treatment of cervically exposed hypersensitive dentin. Am J Dent 25(3): 131-5 [crossref]

\section{Citation:}

Alwael A, Kim I, Steffensen B, Hawley C, Uzel G, et al. (2020) Clinical and Microbiological Outcomes of Er:YAG Laser Used as an Adjunct in Non-Surgical Periodontal Therapy: A Randomized Clinical Trial. J Clin Res Med Volume 4(1): 1-6. 